\title{
Article
}

\section{An exploration of lifestyle/obesity programmes for adults with intellectual disabilities through a realist lens: Impact of a 'context, mechanism and outcome' evaluation}

Taggart, Laurence, Doherty, Alison Jayne, Chauhan, Umesh and Hassiotis, Angela

Available at http://clok.uclan.ac.uk/36255/

Taggart, Laurence, Doherty, Alison Jayne ORCID: 0000-0003-3593-8069, Chauhan, Umesh ORCID: 0000-0002-0747-591X and Hassiotis, Angela (2021) An exploration of lifestyle/obesity programmes for adults with intellectual disabilities through a realist lens: Impact of a 'context, mechanism and outcome' evaluation. Journal of Applied Research in Intellectual Disabilities, 34 (2). pp. 578-593. ISSN 1360-2322

It is advisable to refer to the publisher's version if you intend to cite from the work. http://dx.doi.org/10.1111/jar.12826

For more information about UCLan's research in this area go to http://www.uclan.ac.uk/researchgroups/ and search for <name of research Group>.

For information about Research generally at UCLan please go to http://www.uclan.ac.uk/research/

All outputs in CLoK are protected by Intellectual Property Rights law, including Copyright law. Copyright, IPR and Moral Rights for the works on this site are retained by the individual authors and/or other copyright owners. Terms and conditions for use of this material are defined in the policies page. 


\section{Reflection of lifestyle/obesity programmes in people with intellectual disabilities through a realist lens: impact of a ‘context, mechanism and outcome' evaluation}

\section{Abstract}

Background: Obesity is higher in people with intellectual disabilities.

Aims: There are two aims of this reflective paper. Firstly, using a realist lens, to go beyond 'what works' and examine the 'context, mechanisms and outcomes' (CMO) of lifestyle/obesity programmes for this population. Second, using a logic model framework to inform how these programmes could be implemented within practice.

Method: We explored six review papers and the individual lifestyle/obesity programmes that these papers reviewed using the CMO framework.

Results: There were few theoretically underpinned, multi-component programmes that were effective in the short to long-term and many failed to explore the 'context and mechanisms'. We developed a logic model and engaged in two co-production workshops to refine this model.

Discussion: Using a realist approach, programmes need to be underpinned by theories, multi-component, have a closer understanding of the interplay of the 'context and mechanisms' and delivered using a logic model.

Keywords: intellectual disability, lifestyle/obesity, realist lens, logic modelling 


\section{Reflection of lifestyle/obesity programmes for people with intellectual disabilities through a realist lens: impact of a 'context, mechanism and outcome' evaluation}

\section{Introduction}

Obesity in the general population

Globally, obesity (defined as having a Body Mass Index (BMI) $\geq 30 \mathrm{~kg} / \mathrm{m} 2$ ) is a major health risk for many individuals (Tremmel et al. 2017). It increases an individual's risk of developing a range of medical conditions (co-morbidities) including cardiovascular disease, Type 2 diabetes, certain types of cancer (Wilson et al. 2002; WHO 2004) and premature mortality (WHO 2004; SIGN 2010; NICE 2014). Since 1980, the prevalence of obesity has doubled in at least 70 countries and by 2015, a total of 107.7 million children and 603.7 million adults across the globe were obese (Global Burden of Disease 2015). Internationally, the prevalence of obesity amongst children has been lower than that for adults, but the rate of increase in children with obesity has been greater than the rate of increase in adults with obesity (NICE 2014; Global Burden of Disease 2015). Levels of obesity across low and middle-income countries such as the Middle East, North Africa, Latin America and the Caribbean have approached levels found in higher-income countries (Popkin \& Slining 2013). If these increasing rates continue unchecked, then almost half of the world's adult population will be overweight or obese by 2030 (Dobbs et al 2014). The world-wide economic impact of obesity was estimated to be US $\$ 2.0$ trillion or $2.8 \%$ of the global gross 
domestic product in 2014 (Global Burden of Disease 2015). Obesity-related healthcare costs will pose an even greater economic global burden given the increasing prevalence in both the high income and low-middle income countries in the next century (Wang et al. 2008).

Obesity in people with intellectual disabilities

With an increasing rate of obesity amongst the general population globally, international studies indicate that people with intellectual disabilities are at increased risk of obesity (Ranjan et al. 2018). Data from nationally representative samples of populations with intellectual disabilities are sparse, nevertheless, the prevalence of obesity in adults with intellectual disabilities has increased in countries that have published research in this field (Melville et al. 2007; Stancliffe et al. 2011; Ranjan et al. 2018). Researchers from countries including the UK, The Netherlands, Australia, USA, China, France and New Zealand report that the prevalence of obesity amongst adults with intellectual disabilities is between $17 \%-43 \%$ (Hsieh et al 2014; Mikulovic et al. 2014; Li et al. 2015; Koritsas \& Lacono 2016). In England, obesity affects $38 \%$ of adult patients with intellectual disabilities who are registered with primary healthcare services compared to $30 \%$ of registered adult patients from the general population, who have had their BMI recorded (2016/17) (NHS Digital 2017).

There are several studies that have examined the prevalence of obesity among children and adolescents with intellectual disabilities. Maiano et al. (2016) in the first systematic review and meta-analysis of 16 international studies of this population, 
found that adolescents (aged 12-20 years) with intellectual disabilities to be at greater risk of obesity than their typically developing peers: no comparison studies were found for children ( $<12$ years).

Potential risk factors for people with intellectual disabilities who are obese include: gender (women>men), age (younger>older), cause (i.e. genetic/chromosomal), level of disability, individuals living in less restrictive residential settings, prescription of anti-psychotic medications, poor dietary habits, and very high levels of physical inactivity, sedentary behaviour and poverty (Matthews et al. 2011; Stancliffe et al. 2011; De Winter et al. 2012; Hsieh et al. 2014; Taggart \& Cousins 2014; Hoey et al. 2017; Melville et al. 2018).

People with intellectual disabilities tend to become obese at a much earlier age than the general population (Bhaumik et al. 2008; Melville 2008; PHE 2015), and they are more likely to experience obesity-related medical conditions at a much younger age than the general population such as Type 2 diabetes, coronary heart disease and certain cancers (Melville et al. 2007; Rimmer et al. 2010; Taggart et al. 2013; Cooper et al. 2015). People with intellectual disabilities are more likely to have complex health problems that impact on performing activities of daily living (Hilgenkamp et al. 2014), ability to access and utilise community activities, physical activities, health promotion and screening programmes (Hughes-McCormack et al. 2017) and have a lower quality of life (Pett et al. 2013). Some people may be unknown to health services and therefore may not be offered obesity prevention and weight management multi-component programmes (Taggart et al. 2014). 
People with intellectual disabilities are also more likely to experience inequalities and inequities in healthcare including: communication challenges, a lack of support, discriminatory attitudes by healthcare staff, and a failure by service providers to make 'reasonable adjustments' to meet this populations needs (Michael 2008; Hatton et al. 2011; Ali et al. 2013; Emerson \& Hatton 2014; Carey et al. 2016; Doherty et al., 2020). Whilst some factors (such as genetic/chromosomal conditions) cannot be directly targeted at least for now, lifestyle programmes (i.e. diet/nutrition, physical activity, sedentary behaviour, smoking) can be designed, tailored and delivered to improve health outcomes in this population (Emerson \& Hatton 2014; Marks \& Sisirak 2014; Taggart \& Cousins 2014; Doherty et al. 2017). Tailoring lifestyle/obesity programmes to specific populations can result in ensuring new actions can become normalised into the local context.

\section{Purpose of the reflective paper}

This paper is not a review paper but a reflection of lifestyle/obesity programmes for people with intellectual disabilities, through a realist lens using the 'context, mechanism and outcome' framework as proposed by Pawson \& Tilley (1997). The purpose of this reflective paper is twofold. Firstly, using a realist lens, we explore six literature/integrative/systematic and meta-analysis review papers, and each individual lifestyle/obesity programmes, that have been published for adults with intellectual disabilities using the 'context, mechanism and outcome' framework (Pawson \& Tilley, 1997). This paper goes beyond asking 'what works' to ask, 'what is it about a social [lifestyle/obesity] programme that works for whom, in what 
circumstances, in what respects, over which duration' (Pawson, 2013, p.15). From this learning, our second aim of this paper was to develop a draft logic model that would be able to implement and sustain lifestyle/obesity behaviour change in community settings for people with intellectual disabilities. Through two coproduction workshops with adults with intellectual disabilities and a service organisation, we refined this logic model.

Where lifestyle/obesity programmes are implemented into routine practice based upon this 'context, mechanisms and outcome' model, realist principles have much to offer in reducing obesity and enabling knowledge about longer-term sustainability (Fletcher et al., 2016).

\section{What is realism or realist evaluation?}

'Realism' or 'realist evaluation' is 'underpinned by an understanding of how the world is and how it works (ontology), and an understanding of the nature of knowledge (what we can know and how we can know it (epistemology)' (Emmel et al. 2018, p.43). Traditionally, positive research in the form of experimental studies and randomised controlled trials (RCTs) examine the effects of a social programme on identified outcomes answering the question 'does it work'. These studies generally report the statistical outcomes, in the form of a $p$ value, of whether the programme has caused a specific outcome or not (X leads to $\mathrm{Y}$ ). However, these types of studies have come under criticism from 'realists' (Pawson \& Tilley, 1997; Bhaskar, 2008), as they have failed to understand the programme 'mechanisms' that cause the programme to lead to changes in the identified outcomes. For example, in what 'context' is the social programme delivered in (ie. population, setting, time), and what 
are the 'causal mechanisms' (ie. the underpinning theories of the specific components of the intervention, who is delivering the intervention, resources, fidelity of programme delivery), that makes a programme successful or not, have not been explored within these experimental studies and RCTs. However, more recent RCTs are starting to explore the 'context, mechanism and outcomes' of such complex social programmes. It is beyond the scope of this reflective paper to offer readers a comprehensive explanation of the philosophical foundations of 'realism', but we refer readers to the seminal work of Pawson \& Tilley (1997) and Bhaskar (2008).

Before exploring the 'context, mechanisms and outcomes' of lifestyle/obesity programmes in the intellectual disability population, we will operationally define what is meant by a theoretically informed and multi-component lifestyle/obesity programme for the general population.

\section{Lifestyle/obesity programmes for the general population}

The Medical Research Council $(2000,2008,2019)$ have highlighted the importance of using both individual change theories (ie. Social Cognitive Theory, Theory of Planned Behaviour, Trans-Theoretical Model, Behaviour Change Techniques. etc (Michie et al. 2009)) and system change theories (ie. social ecological model (McLeroy et al. 1988), COM-B model (Michie et al. 2011)) in developing and evaluating complex programmes. Such theories are core in understanding the 'context', 'casual mechanisms', and the process of behaviour change, and 'outcomes' thereby making these theories a fundamental component of any complex 
or lifestyle/obesity programmes. More specifically, SIGN (2010) and NICE (2014) within the UK define successful lifestyle/obesity programmes as requiring four core components (see Table 1). Table 1 summarises these multi-components that focus on a $600 \mathrm{kcal}$ energy deficit diet (EDD), promotion of moderate to vigorous physical activity as per recommendations and use of behaviour change techniques. These theoretically driven and multi-component lifestyle/obesity programmes for the general population have been found to be clinically effective in decreasing weight and improving quality of life in the short and long-term (Loveman et al. 2011; Dombrowski et al. 2012; Avery et al. 2015).

\section{INSERT TABLE I HERE}

\section{Lifestyle/obesity programmes for people with intellectual disability using a realist lens}

Over the past twelve-years there have been six published international reviews of lifestyle/obesity programmes for adults with intellectual disabilities (spanning the time period 1946 to 2019). These include one literature review (Hamilton et al. 2007), two integrative literature reviews (Doherty et al. 2017; Jinks et al. 2010), one systematic review (Spanos et al. 2013) and two combined systematic reviews and metaanalyses (Harris et al. 2018; Willems et al. 2018). We also explored in detail each of these individual lifestyle/obesity programme papers that these six-review paper reviewed (see Table 2). These six international review papers were identified by a brief literature review. The purpose of identifying these review papers was to identify 
the primary research lifestyle/obesity studies for adults with intellectual disabilities. The reviews' included studies that were retrieved and reviewed - including forward citation searches.

\section{INSERT TABLE 2 ABOUT HERE}

Between these six reviews, they have identified 14-individual lifestyle/obesity programmes that are identified as either stand-alone dietary interventions, standalone physical activity interventions, health education/health promotion only interventions, and programmes that have been described as multi-component

programmes (see Table 2). These lifestyle/obesity programme studies have employed qualitative designs, quasi-experiential designs and more recently RCTs. Many of these lifestyle/obesity programme studies have been delivered in a group format whereas others have been delivered on an individual basis. Programmes vary from 6 weeks to 16 -months. There is variation in the primary and secondary outcome measures used across these programmes (i.e. weight, BMI, waist circumference, nutritional knowledge, health knowledge, physical activity, moderate to vigorous physical activity (MPVA), etc.), making comparison difficult.

\section{Examining 'context, mechanisms and outcomes'}

From Table 2 it can be observed that some of these studies have reported limited weight loss in the short-term. None of these papers have reported significant weight loss in the long-term. Many of these earlier lifestyle/obesity programmes have small 
samples, have not been powered, do not have a control group, lack clarity in the frequency and dosage of the behaviour change techniques employed, and do not have long-term follow-up (Hamilton et al. 2007; Jinks et al. 2010; Spanos et al. 2013; Doherty et al. 2017; Harris et al. 2018; Willems et al. 2018). Although more recent lifestyle/obesity programmes for adults with intellectual disability are using a RCT design that have addressed these methodological and practical challenges of the earlier programmes, some of these studies continue to report increase in physical activity and limited weight loss in the short-term (Spanos et al. 2013; Doherty et al. 2017; Harris et al. 2018; Willems et al. 2018). However, none of the studies reported significant reduction in obesity in the long-term. Therefore, it is important to ask why are these lifestyle/obesity programmes not successful for this population? It is here where we use the realist lens using the 'context, mechanisms and outcomes' framework as proposed by Pawson \& Tilley (1997) to explore these lifestyle/obesity programmes in further detail.

In order to achieve both significant short and long-term weight loss (5-10\%), all lifestyle/obesity programmes need to be theoretically underpinned (MRC, 2000, 2008, 2019) and comprise several multi-components whether for adults with or without disabilities (SIGN 2010; NICE 2014). Harris et al. (2018) in their systematic review and meta-analysis of six RCTs of lifestyle/obesity programmes for adults with intellectual disabilities, reported that these programmes were not multi-component as detailed, as these six programmes did not strictly adhere to the clinical recommendations as identified by NICE (2014) and SIGN (2010) to facilitate weight loss (see Table 1). 
Another core component in reducing weight is the use of behaviour change techniques (i.e. goal setting, self-monitoring and providing feedback on performance) (NICE 2014; SIGN 2010). Harris et al. (2018) found only self-monitoring of behaviour was frequently used across these six lifestyle/obesity programme studies. Behaviour change may be challenging for some individuals with intellectual disabilities who may lack social support. The authors highlighted the complexity for adults with intellectual disabilities (cognitive impairments, communication deficits, lack of control, lack of motivation, dependence on others) and their carers (i.e. motivation, commitment, role models, etc.) in fully engaging with a 6-18-month lifestyle/obesity intervention.

Doherty et al. (2017) in their integrative review found only two tailored lifestyle/obesity multi-component programme for adults with intellectual disabilities to be effective in reducing obesity in the short-term: 'Take 5 ' in Scotland (Melville et al 2011; Spanos et al. 2014; Harris et al. 2017; 2019;) and a lifestyle/obesity multicomponent programme developed in Sweden (Bergstrom et al 2013; Sundholm et al. 2015). Both programmes were underpinned by individual change theories (Social Cognitive Theory and the trans-theoretical model respectively). 'Take 5' is based upon a personalised 1-1 programme with themed sessions delivered by motivated and supportive carers. The Swedish programme is delivered within a group format in residential homes.

Willems et al. (2018) undertook a further systematic review and meta-analysis of eight RCTs of lifestyle/obesity change programmes for people with intellectual disabilities. Four studies used physical activity only (Curtin et al. 2013; Melville et al. 2015; Shields \& Taylor 2015; Boer \& Moss 2016) and four studies used physical activity and nutrition together (McDermott et al. 2012; Beeken et al. 2013, 
2015; Marks et al. 2013): all these programmes were tailored to this population. Five of the eight studies used Social Cognitive Theory as the programme's theoretical framework. This review found that these lifestyle/obesity programmes for this population 'have a small, though, positive effect on lifestyle, as demonstrated by meta-analyses on BMI, weight, and waist circumference, however, the effects were only statistically significant for waist circumference' and in the short-term (p. 957). However, such programmes have not been optimally tailored by staff to meet the needs of people with intellectual disabilities and the fidelity of such programmes has been questioned.

Caution must be taken in interpreting the findings of these individual and review papers given the different types of lifestyle/obesity programmes identified, designs employed, the methodological limitations that constrain the strength of the evidence, and limited increase in physical activity and reduction in weight in the short-term. No studies reported long-term increases in physical activities and obesity reduction. Some of the lifestyle/obesity programmes did not identify any theoretical underpinning whilst others identified using only one individual change theory, mainly based on Social Cognitive Theory. Many of the lifestyle/obesity programme studies' authors did not provide detailed information on the 'context' and 'mechanisms' of the interventions. Some programmes do go beyond the individual change level that may impact on its implementation (ie. support from an interdisciplinary group of professionals (organisational level), identification of what type of exercise will work where (community level), carer support groups, involvement of staff in developing the programme) (see Table 2). However, some studies only measured change at the individual level. 
The authors of these review papers, and the individual lifestyle/obesity programme papers, have not explored the role of the interplay of 'context, mechanisms and outcomes' in which these programmes were developed and evaluated. Furthermore, there has been little research undertaken to understand why such lifestyle/obesity programmes for this population are not implemented and sustained within community settings.

Individual barriers to changing lifestyle/obesity behaviour

Individual behaviour change may be difficult for people with intellectual disabilities who may not have the comprehension, competence or motivation to engage with, or maintain, positive lifestyle behaviour choices. Individual education and motivation are not enough to sustain behaviour change for this population given their range of cognitive impairments and communication difficulties (Brehmer-Rinderer et al. 2013; Owens et al. 2020). People with intellectual disabilities have a greater reliance upon their family/paid carers to make informed decisions pertaining to healthy lifestyle choices (ie. reduction in fatty and processed foods, reduction in sedentary behaviour, increase in physical activity, reduction in smoking, etc) (Taggart \& Cousins 2014; Owens et al. 2020). However, these carers may not be fully informed and empowered to support the person with intellectual disabilities to make these healthy lifestyle choices (Hanna et al. 2011). Willems et al. (2018) suggest that many of the current lifestyle/obesity programmes are not optimally adapted or tailored for this population. There are also concerns over the applicability of complex behaviour change techniques for people with intellectual disabilities (Willems et al. 2018). 
Systems or organizational barriers to behaviour change

The individual with an intellectual disability and their family/paid carers are central in how successful a lifestyle/obesity programme is effective in the short and long-term, but, as this paper is highlighting, the 'context' and 'mechanisms' of how such programmes are developed and delivered are also fundamental for its success.

There is growing evidence of the challenges that systems, organisations and cultures have in restricting lifestyle/obesity behaviour change:

- Limited health promotion culture within the organisation (reactive approach to health problems, a lack of health promotion guidelines/policies, limited health promotion priorities, a lack of awareness of health education/training)

- A lack of health promotion capacity within the organisation (lack of staff empowerment and motivation, limited external health promotion support, a lack of organisational commitment to health programme delivery, a lack of a budget, resources and time)

- Poor communication between management and front-line staff (a lack of meetings, poor communication systems) and

- A lack of partnerships with communities/universities that may prevent staff from supporting individuals with intellectual disabilities from engaging in healthy behaviours (Naaldenberg et al. 2013; Casey et al. 2015; Spassiani et al. 2016; 2019; O'Leary et al. 2018; Owens et al., 2020). 
It is now being strongly argued that organizational support for behaviour change is particularly significant to effectively sustain behaviour change, increase physical activity and weight reduction in the short to long-term (Spassiani et al. 2016; 2019; Kuijken et al. 2015; O’Leary et al. 2018; Owens et al. 2020).

Recognising the interplay of the 'context' and 'mechanisms' within the complex system

There is a growing body of research that now recognises that lifestyle/obesity programmes are not easily being implemented within organizations/clinical practice: for both the general and intellectual disability populations. There has been little recognition of the interplay of the 'context' and 'mechanisms' in the implementation of these lifestyle/obesity programmes (Pawson \& Tilley 1997; Fletcher et al. 2016). It is now being strongly purported that programmes themselves are not necessarily complex, but it is the organization and surrounding systems that are complex, and therefore, there is a greater need to consider how to implement these lifestyle/obesity programmes in such complex organizations and systems (Moore et al. 2012, 2019; Jamal et al. 2015; Moore \& Evans 2017).

Moore \& Evans (2017) stated that this emphasis on underpinning programmes by individual change theories has been at the cost of overlooking the 'mechanisms through which a problem is actually sustained'. Moore \& Evans (2017) further highlighted that closer attention is required pertaining to the 'context, and the ecological fit of programmes with the systems whose functioning they attempt to change'. 
Implementing lifestyle/obesity programmes without recognising the interplay of the 'context' and the 'mechanisms' of change within the organization, can be purported to be too simplistic and will not lead to significant reductions in obesity. This is evidenced by the continued reporting of high levels of obesity in young people and adults with intellectual disabilities, and the lack of up-take of lifestyle/obesity programmes as highlighted above in these six review papers (Hamilton et al. 2007; Jinks et al. 2010; Spanos et al. 2013; Doherty et al. 2017; Harris et al. 2018; Willems et al. 2018). Having a clearer knowledge of how the problem (i.e. obesity) has developed, and it is sustained, is therefore required; as well these lifestyle/obesity programmes being underpinned by both individual change theories (i.e. SCT, trans-theoretical model) and system change theories (i.e. socio-ecological model, COM-B).

This must be accompanied together with a greater understanding of the 'context' and 'mechanisms' of change within which these lifestyle/obesity programmes have to be applied within community settings to achieve weight reduction (Bonell et al. 2012; Moore et al. 2012, 2019; Craig et al. 2015; Hawe 2015; Jamal et al. 2015; Spassianai et al. 2016, 2019; Moore \& Evans, 2017; O'Leary et al. 2018; Owens et al. 2020). 'Context' and 'mechanisms' have rarely been explored within the field of obesity and intellectual disability.

Pfadenhauer et al. (2017) developed the 'Context and Implementation of Complex Programmes ( $\mathrm{ClCl}$ ) framework' comprising of three dimensions to examine the context and system: 
- Context consists of geographical, epidemiological, socio-cultural, socioeconomic, ethical, legal, political;

- Implementation consists of implementation theory, process, strategies, agents and outcomes;

- Setting refers to the specific organization in which the programme is put into practise.

The effectiveness of any lifestyle/obesity programme is dependent upon the 'context' within which it is implemented. Despite many positive RCTs of lifestyle/obesity interventions, many do not target the 'context', 'mechanisms' and implementation in a combined approach: hence why so many lifestyle/obesity programmes fail to sustain longer-term behaviour change. The $\mathrm{CICl}$ framework provides a 'structured and comprehensive conceptualisation and assessment of the setting, context and implementation of complex interventions' (Pfadenhauer et al. 2017, p.1). This framework can support researchers and clinicians to observe how lifestyle/obesity programmes can be scaled-up and implemented with greater success across a range of settings.

\section{Implementing lifestyle/obesity interventions: using a logic model}

The second goal of this paper was to apply this contemporary thinking to lifestyle/obesity management in adults with intellectual disabilities by proposing a logic model framework. A logic model is a systematic and an effective visual 'description of a system, designed to identify important elements and 
relationships within that organization' (WG Kellogg, 2014). The goal of a logic model is to present a clear plan for the utilization of resources to meet the identified goals/objectives. Involving all relevant stakeholders who have a clear understanding of the context, system and organization in a series of co-production workshops that can alter the mechanisms that support the delivery of lifestyle/obesity programme (Anderson 2010). Logic models can also be used to evaluate the effectiveness of the programmes and organisations. A logic model can provide an understanding of:

- The context and system (i.e. physical environment, resources, who are the main stakeholders, perception of the problem by all the stakeholders, opportunity versus lost costs, legal requirements for addressing the problem, ethical issues, the political and policy climate for reaching a tipping point, etc.)

- The inputs/resources (i.e. human, financial, organizational, community resources, etc.) that are needed to deliver the $\mathrm{MCl}$

- The outputs/activities (i.e. processes, tools, events, technology, actions, etc.) that are needed to be put in place to deliver the $\mathrm{MCl}$ and sustain the change; and

- The short to medium outcomes (3-10 years) (i.e. changes in behaviour, knowledge, attitudes, etc.) and the longer-term impacts at the system level (10-20 years).

There is an evolving literature that is now exploring our understanding of the 'context, mechanism and outcomes' that 'can promote the integration of policies, supports and outcomes within the field of intellectual disabilities': suggesting logic models be utilized within organizations (Isaacs et al. 2009; Shogren et al. 2015, 
2018; Claes et al. 2017). The use of logic models is a relatively novel approach in examining the 'context, mechanisms and outcomes' within organizations to implement and sustain behaviour change in the field of intellectual disabilities. A small number of logic models have been developed that have targeted improving the quality of life (Scholack et al. 2010) and diminishing challenging behaviours, via Positive Behaviour Support (Scott et al. 2018), for the intellectual disability population. Harris et al. (2019) in their process evaluation paper of the programme 'Take 5' for adults with intellectual disabilities, presented an obesity logic model. This model was based upon WG Kellogg Foundation (2014) highlights the inputs, programme components, behaviour outputs and outcomes.

\section{Insert Figure 1 about here}

Theory driven logic models are currently being utilised to underpin programme developments and as part of the requirement for national research funders (ie. National Institute of Health Research (NIHR) in the UK). Peyton \& Sicchitano (2017) have argued that despite the co-production with stakeholders in developing and refining such logic models and testing in a research setting, they then remain 'abandoned' and redundant in community settings; and not used as a practical and useful framework to guide implementation. In further developing the logic model we embed the $\mathrm{CICl}$ framework as identified above (Pfadenhauer et al. 2017), to examine the 'context mechanisms and outcomes' of an organization for 
implementing, and sustaining, lifestyle/obesity programmes for people with intellectual disabilities.

In Figure 1 we have advanced and provided a detailed logic model that graphically details the integrated context, systems, resources, activities, mechanisms of change, outputs, outcomes and impact of an lifestyle/obesity programmes for adults with intellectual disabilities, that could lead to effective implementation of weight reduction within community organizations.

\section{Refinement of the logic model}

This logic model was developed by the authors to support the conceptualization, organization and planning of a lifestyle/obesity programme (ie. context, inputs, outputs, mechanisms and outcomes). Two co-production workshops were used to refine the logic model with relevant stakeholders, this has been strongly recommended by the MRC $(2000,2008,2019)$ and is also consistent with the realist paradigm. Purposeful sampling was used to recruit participants with the relevant knowledge, skills and experience. One workshop was held with the organization's Chief Executive, one Family Services Manager, two Operational Managers and eight Service Managers) within one large regional service organisation for children and adults with intellectual disabilities in $\mathrm{N}$ Ireland. Another workshop was held with a group of four adults with mild intellectual disabilities and one member of staff from a local advocacy organisation. The workshops were designed according to the principles for successful co-production identified by Greenhalgh et al. (2016): there were clear aims for each workshop, they were made as practical as possible and 
involved a combination of presentations, small group work and discussions amongst the whole group together. The data were collected via flip-chart materials and field notes. The first author facilitated these two workshops.

With staffs' support from the local advocacy organisation, we had to ensure that the complex terminology used within the logic model, and each section, was simplified and carefully explained. Overall, the concept of implementing a lifestyle/obesity programme and using a logic model was viewed as positive, the adults with intellectual disabilities liked the idea of it being multi-component and reported that this approach would be more successful. There was discussion about the components of the 'Take 5' intervention, the length, recommended physical activity goals and that the 6 follow-up sessions. The group liked the concept of a personalised focused 1-1 programme and highlighted the importance of the involvement of parents/carers in supporting them with the intervention. The service users asked that a person with an intellectual disability be a co-trainer within the delivery of the lifestyle/obesity programme. The logic model was refined.

The workshop with the regional service organisation for children and adults with intellectual disabilities was received very well. One of the Operation Managers reported 'this is a perfect model for delivering strategic outcomes, within our organization and also in statutory Health \& Social Care organisations ... pulling together governance (who can make that change happen), mechanisms for change and short/medium/long-term outcomes'. There was some discussion about some of the inputs, outputs, mechanisms and the model was refined slightly. The senior managers were very positive about the logic model targeting lifestyle/obesity but also reported that it 'we [the organization] should adopt this for all of our strategies in the 
Business Plan and/or it could lend itself to being adopted for projects at different outcomes at different stages of growth 'Pilot', 'Regional' and 'National' deployment.'

\section{Strengths and limitations}

This paper was underpinned by a realist lens within which these theoretically underpinned and multi-component lifestyle/obesity programmes have been viewed as events within 'complex systems' (Moore \& Evans 2017; Moore et al. 2019). From this perspective, these lifestyle/obesity programmes are understood as the interaction between their 'context, mechanisms and outcomes' (Pawson \& Tilley 1997). A major strength of this paper has been the engagement with adults with intellectual disabilities and senior management within a specific organization as service recipients in the refining of the logic model. This study makes a unique contribution to the field of intellectual disability and lifestyle/obesity, programme development and translating research into practice. This paper highlights the need for more research into developing social programmes that utilise both individual and system change theories, 'context, mechanisms and outcomes' and incorporating coproduction workshops with key stakeholders that directly result in the development and refinement of a logic model.

Future evaluative reviews should not only examine the clinical and cost-effectiveness of any intellectual disability social programmes but also undertake a realist systematic review and a meta-analyses to explore the implementation parameters within the 'context, mechanism and outcomes' using the RAMSES framework (Pawson \& Tilley 1997; Hawe 2015; Moore \& Evans 2017; Moore et al. 2019). This 
will also include qualitative studies, policy papers, professional guidelines etc. Managers and front-line staff may benefit from training on realist principles, including 'context, mechanisms and outcomes' configurations, and systems thinking (Pawson \& Tilley 1997; Moore et al., 2019). This may support them to understand how their decision-making is influenced by 'contextual' factors and encourage reflection on the 'mechanisms' underpinning their practice which may, in turn, promote evidence-based practice ('outcomes').

This reflective paper was limited to review and the individual programme papers only, English language studies, focusing only on adults with intellectual disabilities. We did not examine papers that identified children with intellectual disabilities and other cognitive disability populations such as those with Autism Spectrum Disorder, were there may be transferable evidence. We developed and refined a logic model within this paper as the proposed framework for translating the evidence into practice. We acknowledge there are other frameworks that could offer researchers and practitioners other mediums for this knowledge translation, but these have not been explored due to word restrictions.

\section{Conclusion}

Obesity is a global health and social problem with dramatically increasing rates largely due to the influences of the obesogenic environment (through their impact on physical activity and dietary behaviours at individual and community levels) (Swindburn et al. 2011). People with intellectual disabilities are at a higher risk to such influences and face a greater burden of related diseases and 
complications. We only found two theoretically underpinned, evidenced-based multicomponent lifestyle/obesity programmes for people with intellectual disabilities.

Current evidence would suggest lifestyle/obesity programmes are likely to have the greatest impact in supporting people with intellectual disabilities to manage their weight: but these lifestyle/obesity programmes are not being translated into clinical practice. Programmes focused on the 'context, mechanisms and outcomes' requires collaboration with health and social care settings, local authorities and planning departments. The proposed logic model articulates the multi-factorial determinants of increased risk for people with intellectual disabilities in relation to obesity reduction. We propose that a logic model that graphically presents context, systems, resources, activities, mechanisms of change, outputs, outcomes and impact, can help successfully implement and sustain such programmesfor this population in local communities. Where lifestyle/obesity programmes are implemented into routine practice, realist principles have much to offer in reducing obesity and enabling knowledge about longer-term sustainability. 
Acknowledgements: Dr. Alison Doherty was funded by the National Institute for Health Research (NIHR) Applied Research Collaboration North West Coast (ARC NWC). The views expressed in this publication are those of the authors and not necessarily those of the NHS, the NIHR, or the Department of Health and Social Care. 


\section{References}

Ali A, Scior K, Ratti V, Strydom A, King M, Hassiotis A. (2013) Discrimination and other barriers to accessing health care: perspectives of patients with mild and moderate intellectual disability and their carers. PLOS ONE. 8(8):e70855.

Anderson L. (2010) Using logic models to capture complexity in systematic review. Research Synthesis Methods; 2(1); 33-42.

Avery L, Flynn D, Dombrowski SU, Wersch A, Sniehotta FF, Trenell MI (2015) Successful behavioural strategies to increase physical activity and improve glucose control in adults with type 2 diabetes. Diabetic Medicine. 32(8):1058-1062.

Beeken RJ, Lally P, Wahlich C, Mar R, Marston L, Wilson R. et al. (2015) Piloting a manualised weight management programme for overweight and obese persons with mild-moderate learning disabilities: Results of the shape up-LD study. Obesity Facts, 8 (Suppl.1): 197.

Beeken RJ, Spanos D, Hunter R, Hassiotis A, King M, Omar R. et al. (2013) Shape up-LD: A pilot randomised controlled trial of a manualised weight management programme for adults with mild-moderate learning disabilities. Obesity Facts, 6:192 Bergstrom H, Hagstromer M, Hagberg J, \& Elinder LS. (2013) A multi-component universal programme to improve diet and physical activity among adults with intellectual disabilities in community residences: A cluster randomised controlled trial. Research in Developmental Disabilities. 34(11): 3847-3857.

Bhaumik S, Watson JM, Thorp CF, Tyrer F, McGrother CW (2008) Body mass index in adults with intellectual disability: Distribution, associations and service 
implications: A population-based prevalence study. Journal of Intellectual Disability Research. 52(4): 287-298.

Boer PH, Moss, SJ. (2016) Effect of continuous aerobic vs interval training on selected anthropometrical, physiological and functional parameters of adults with Down syndrome. Journal of Intellectual Disability Research. 60(4): 322-334.

Bonnell C, Fletcher A, Morton M, Lorenc T \& Moore L. (2012) Realist randomised controlled trials: a new approach to evaluating complex public health interventions. Soc Sci Med; 75(12); 2299-306

Brehmer-Rinderer, B, Zigrovic L, Naue U, Weber G. (2013) Promoting the health of persons with intellectual disabilities using the UN Convention on the rights of persons with disabilities: Early implementation assessment in Spain and Hungary. Journal of Policy and Practice in Intellectual Disabilities. 10(1):25-36.

Campbell M, Fitzpatrick R, Haines A, Kinmonth AL, Sandercock P, Spiegelhalter D. et al. (2000) Framework for the design and evaluation of complex programmesto improve health. BMJ; 321:694-6.

Carey IM, Shah SM, Hosking FJ. et al. (2016) Health characteristics and consultation patterns of people with intellectual disability: a cross-sectional database study in English general practice. British Journal of General Practice. 66(645):e264-e270.

Casey MM, Payne WR, Eime RM. (2015) Organisational readiness and capacity building strategies of sporting organisations to promote health. Sport Management Review. 15(1): 109-124, 
Claes C, Ferket H, Vandevelde S, Verlet D, De Maeyer J. (2017) Disability policy evaluation: combing logic models and systems thinking. Intellectual and Developmental Disabilities; 55(4); 247-257.

Cooper SA, McLean G, Guthrie B. et al. (2015) Multiple physical and mental health comorbidity $\mathrm{n}$ adults with intellectual disabilities: population-based cross-sectional analysis. BMC Family Practice. 16:110.

Craig FM, Audrey, S, Barker M, Bond L, Bonnell C, Hardeman W, Moore L, et al. (2015) Process evaluation of complex interventions. Medical Research Council.

Craig P, Dieppe P, Macintyre S, Michie S, Nazareth I. Pettigrew M. (2008) Developing and evaluating complex interventions: the new Medical Research Council guidance. BMJ. Sep 29; 337:a1655. doi: 10.1136/bmj.a1655.

Curtin C, Bandini LG, Must A, Gleason J, Lividini K, Phillips S. et al. (2013) Parent support improves weight loss in adolescents and young adults with Down syndrome. The Journal of Pediatrics. 163(5):1402-1408.

de Winter CF, Bastiaanse LP, Hilgenkamp TI, Evenhuis HM, Echteld, MA. (2012) Overweight and obesity in older people with intellectual disability. Research in Developmental Disabilities. 33(2): 398-405.

Dobbs R, Sawers C, Thompson F, et al. (2014) Overcoming Obesity: An Initial Economic Analysis. McKinsey Global Institute: Jakarta, Indonesia.

Doherty AJ, Jones SP, Chauhan U, Gibson JME. (2017) An integrative review of multicomponent weight management programmesfor adults with intllectual difficulties. J Appl Res Intellect Disabil. 2017;(0)1-13. 
Doherty AJ, Atherton, H., Boland, P., Hastings, R., Hives, L., Hood K., JamesJenkinson, L., Leavey, R., Randell, E., Reed, J., Taggart L., Wilson, N., Chauhan, U., (2020) Barriers and facilitators to primary health care for people with intellectual disabilities and / or autism: an integrative review. BJGP Open https://doi.org/10.3399/bjgpopen20X101030

Dombrowski SU. Sniehotta FF, Avenell A, Johnston M, MacLennan G, AraujoSoares V. (2012) Identifying active ingredients in complex behavioural programmesfor obese adults with obesity-related co-morbidities or additional risk factors for co-morbidities: A systematic review. Health Psychology Review. 6(1):732.

Emerson E, Hatton C. (2014) Health Inequalities and People with Intellectual Disabilities. Cambridge University Press. United Kingdom.

Fisher E. (1986) Behavioral weight reduction program for mentally retarded adult females. Perceptual \& Motor Skills. 62(2): 359-362.

Fletcher A, Jamal F, Moore GF, Evans RE, Murphy S. \& Bonell C. (2016) Realist complex programme science: applying realist principles across all phases of the MRC framework for developing and evaluating complex interventions. Evaluation: 22(3), 286-303.

Fox RA, Haniotes H, Rotatori A. (1984) A streamlined weight loss program for moderately retarded adults in a sheltered workshop setting. Applied Research in Mental Retardation. 5(1): 69-79. 
Global Burden of Disease (2015) Global, regional, and national life expectancy, allcause mortality, and cause-specific mortality for 249 causes of death, 1980-2015: a systematic analysis for the Global Burden of Disease Study 2015. Lancet. 8;388(10053): 1459-1544.

Greenhalgh T, Jackson C, Shaw S. \& Janamian T. (2016) Achieving research impact through co-creation in community-based health services: literature review and case study. The Milbank Quarterly, 94, 392-429.

Hanna LM, Taggart L. Cousins W. (2011) Cancer Prevention and Health Promotion for People with Intellectual Disabilities: An Exploratory Study of Staff Knowledge. Journal of Intellectual Disability Research, 55(3): 281-191.

Harris L, Hankey C, Jones N. et al. (2017) A cluster randomised control trial of a multi-component weight management programme for adults with intellectual disabilities and obesity. British Journal of Nutrition. 118: 229-240.

Harris L, Hankey C, Jones N. et al. (2018b) Process evaluation of a clusterrandomised controlled trial of multi-component weight management programme in adults with intellectual disabilities and obesity. Journal of Intellectual Disability Research. Doi.10.111/jir.12563.

Harris L, Melville CA, Murray H, Hankey C. (2018a) The effects of multi-component weight management programmeson weight loss in adults with intellectual disabilities and obesity: A systematic review and meta-analysis of randomised controlled trials. Research in Developmental Disabilities. 72: 42-55. 
Hatton C, Roberts H, Baines S. (2011) Reasonable Adjustments for People with Learning Disabilities in England: A National Survey of NHS Trusts. Improving Health and Lives: Learning Disabilities Observatory, Durham.

Hawe P. (2015) Lessons for complex programmesto improve health. Annual Review Public Health. 18(36):307-323.

Hilgenkamp TI, van Wijck R, Evenhuis HM. (2014) Subgroups associated with lower physical fitness in older adults with ID: results of the HA-ID study. Res Dev Disabil. 35(2): 439-447.

Hoey E, Staines A, Walsh D, Corby, D, Bowers K, Belton S. et al. (2017) An examination of the nutritional intake and anthropometric status of individuals with intellectual disabilities: Results from the SOPHIE study. Journal of Intellectual Disabilities. 21:346-365.

Hsieh K, Rimmer JH, Heller T. (2014). Obesity and associated factors in adults with intellectual disability. Journal of Intellectual Disability Research. 58(9): 851-863.

Hughes-McCormack LA, Rydzewska E, Henderson, A. et al (2017) Prevalence of mental health conditions and relationship with general health in a whole-country population of people with intellectual disabilities compared with the general population. British Journal of Psychology Open. 3: 243-248.

Isaacs B, Clark C, Correia S, Fannery J. (2009) Using logic models to plan quality of life outcome evaluations. Journal of Policy \& Practice in Intellectual Disabilities; 6(1)2-6. 
Jamal F, Fletcher A, Shakleton N, Elbourne D, Viner R, Bonell C (2015) The three stages of building and testing mid-level theories in a Realist RCT: a theoretical and methodological case-example. Trials. 16: 466.

Kellogg Foundation W.K. (2004) Logic Model Development Guide. Battle Creek, MI: W.K Kellogg Foundation.

Koritsas S. Lacono T. (2016) Weight, nutrition, food choice, and physical activity in adults with intellectual disability. Journal of Intellectual Disability Research. 60(4): 355-364

Kuijken NMJ, Naaldenberg J, Nijhuis-van der Sanden MW. \& van Schrojenstein Lantman de Valk H. (2015) Healthy living according to adults with intellectual disabilities: towards tailoring health promotion initiatives. Journal of Intellectual Disability Research. 60(3): 228-241.

Li H, Frey GC, McCormick BP, Johnston JD. (2015) Comparison of obesity among Chinese and US Special Olympic athletes with intellectual disabilities. Research in Developmental Disabilities. 41: 94-100.

Loveman E, Frampton GK, Shepherd J. et al. (2011) The clinical effectiveness and cost-effectiveness of long-term weight management schemes for adults: a systematic review. In: NIHR Health Technology Assessment programme: Executive Summaries. Southampton (UK): NIHR Journals Library; 2003-

.Available from: https://www.ncbi.nlm.nih.gov/books/NBK56859/

Maiano C, ue O, Morin AJS, Moullec G. (2016) Supporting information for Prevalence of overweight and obesity among children and adolescents with 
intellectual disabilities: a systematic review and meta-analysis. Obesity Reviews. Vol 17(7): 7599-611.

Marks B, Sisirak J. (2014) Health promotion and people with intellectual disabilities. In L. Taggart \& W. Cousins (Eds.) Health promotion for people with intellectual and developmental disabilities (pp. 17-29). Maidenhead, UK: Open University Press/McGraw-Hill Publishers.

Marks B, Sisirak J. Chang Y. (2013) Efficacy of the HealthMatters Program Trainthe-Trainer Model. Journal of Applied Research in Intellectual Disabilities. 26(4): 319-334.

Matthews L, Penpraze V, Boyle S. et al. (2011) Agreement of accelerometer and a physical activity questionnaire in adults with intellectual disabilities. Preventative Medicine. 52(5): 361-264.

McDermott S, Whitner W, Thomas-Koger M, Mann JR, Clarkson J, Barnes L. et al. (2012) An efficacy trial of 'steps to your health', a health promotion programme for adults with intellectual disability. Health Education Journal. 71(3): 278-290.

McLeroy KR, Bibeau D, Steckler A, Glanz K. (1988) An ecological perspective on health promotion programs. Health Education and Behaviour; 15: 351-377.

Meldrum DR, Morris MA, Gambone JC. Obesity pandemic: causes, consequences, and solutions-but do we have the will? Fertil Steril. 2017;107(4):833-839.

doi:10.1016/j.fertnstert.2017.02.104 
Melville C, Hamilton S, Hankey CR, Miller S, Boyle S. (2007) The prevalence and determinants of obesity in adults with intellectual disabilities. Obesity Reviews. 8: 223-230.

Melville C, McGarty A, Harris L, Hugest-McCormack L, Baltzer M, McArthur M. et al. (2018) A population-based, cross-sectional study of the prevalence and correlates of sedentary behaviour of adults with intellectual disabilities. Journal of Intellectual Disability Research. 62: 60-71.

Melville CA, Boyle S, Miller S, Macmillan S, Penpraze V, Pert C. et al. (2011) An open study of the effectiveness of a multi-component weight loss programme for adults with intellectual disabilities and obesity. British Journal of Nutrition. 105(1): 1553-1562.

Melville CA, Cooper SA, Morrison J, Allan L, Smiley E, Williamson A. (2008) The prevalence and determinants of obesity in adults with intellectual disabilities. Journal of Applied Research in Intellectual Disabilities. 21: 427-437.

Melville CA, Mitchell F, Stalker K, Matthews L, McConnachie A, Murray HM et al. (2015) Effectiveness of a walking programme to support adults with intellectual disabilities to increase physical activity: Walk well cluster-randomised controlled trial. International Journal of Behavioral Nutrition and Physical Activity. 12(1): 1-11.

Michael J. (2008) Healthcare for all: Report of the independent inquiry into access to healthcare for people with intellectual disabilities. London. Department of Health. 
Michie S, Abraham C, Whittington C, McAteer J, Gupta S. (2009) Effective techniques in healthy eating and physical activity interventions: A metaregression. Health Psychology. 28(6): 690.

Michie, S., Van Stralen, M., and West, R. (2011) The behaviour change wheel: A new method for characterising and designing behaviour change interventions. Implementation Science, 6, pp. 42.

Mikulovic J, Vanhelst J, Salleron T, et al. (2014) Overweight in intellectually-disabled population: Physical, behavioral and psychological characteristics. Research in Developmental Disabilities. 35(1): 153-161.

Moore G, Evans R. (2017) What theory, for whom and in which context? Reflections on the application of theory in the development and evaluation of complex population health interventions. SSM Population Health. 3:132-135.

Moore GF, Audrey S, Barker M, Bond L, Bonell C, Hardeman W. et al. (2012) Process evaluation of complex interventions: Medical Research Council guidance. BMJ; 350:h1258

Naaldenberg J, Kuijken N, van Dooren K, van Schrojenstein Lantman de Valk H. (2013) Topics, methods and challenges in health promotion for people with intellectual disabilities: A structured review of literature. Research in Developmental Disabilities. 34(12): 4534-4545.

National Obesity Observatory (2010) Obesity and life expectancy: Briefing Note. http://webarchive.nationalarchives.gov.uk/20170110170044/https://www.noo.or g.uk/NOO_about_obesity/obesity_and_health [accessed 02/10/19] 
NHS Digital (2017) Health and Care of People with Learning

Disabilities. https://digital.nhs.uk/data-and-information/data-tools-and-services/dataservices/general-practice-data-hub/health-and-care-of-people-with-learningdisabilities [accessed 20/11/19].

NICE (National Institute for Health and Care Excellence) Clinical Guidance PH53 (2014) Managing Overweight and Obesity in Adults - Lifestyle Weight Management Services. Issued May 2014. http://www.nice.org.uk/guidance/ph53 [accessed 20/10/19].

O'Leary, L, Taggart, L, Cousins, W. (2018) Healthy lifestyle behaviours for people with intellectual disabilities: An exploration of organizational barriers and enablers. J Appl Res Intellect Disabil. ; 31(Suppl. 1): 122-135. https://doi.org/10.1111/jar.12396 Owens R, Earle S, McNulty C \& Tilley E. (2020) What works in community health education for adults with learning disabilities: A review of the literature. Journal of Applied Research in Intellectual Disabilities. Doi: 10.111/jar.12746

Pawson R. Tilley N. (1997) Realistic evaluation. London: Sage.

Pett M, Clark L, Eldredge A. et al. (2013) Effective healthy lifestyle change in overweight and obese young adults with intellectual disability. American Journal on Intellectual and Developmental Disabilities. 118(3): 224-243.

Peyton DJ, Sicchitano M. (2017) Devil is in the details: Using logic models to investigate program process. Evaluation and Programme Planning: 65, 156-162. 
Pfadenhauer L, Gerhardus A, Mozygemba K, Lysdahl KB, Booth A, Hofmann B, et al. (2017) 'Context and Implementation of Complex Programmes(CICl) framework.' Implementation Science: 12:21

Popkin BM, Slining MM. (2013) New dynamics in global obesity facing low-and middle-income countries. Obesity Reviews. 14(S2): 11-20.

Public Health England, (2015) Obesity and Health. http://webarchive.nationalarchives.gov.uk/20170110170044/https://www.noo. org.uk/NOO_about_obesity/obesity_and_health [accessed 02/010/19].

Ranjan S, Nasser JA, Fisher K. (2018) Prevalence and potential factors associated with overweight and obesity status in adults with intellectual developmental disorders. Journal of Applied Research in Intellect Disabilities.

Rimmer JH, Yamaki K, Lowry D, Wang E, Vogel LC. (2010) Obesity and obesityrelated secondary conditions in adolescents with intellectual / developmental disabilities. Journal of Intellectual Disability Research. 54(9): 787-794.

Salvador-Carulla L, Reed G, Vaez-Azizi LM, Cooper SA, Martinez-Leal R, Bertelli M. et al. (2011) Intellectual developmental disorders: towards a new name, definition and framework for "mental retardation/intellectual disability" in IC11. World Psychiatr. 10(3): 175-180.

Scholack RL, Keith, KD, Verdugo MA, Gomez LE. (2010) Quality of life model development and use in the field of intellectual disability. In: Kober R. (eds) Enhancing the quality of life of people with intellectual disabilities. Social Indicators Research Series, Vol 41; 17-21. 
Scott SJ, Denne LD, Hastings RP. (2018) Developing a logic model to guide evaluation of impact for learning disability projects: the case of the Positive Behavioural Support (PBS) Academy. Tizard Learning Disability Review: 23(3); 125-132.

Shields N, Taylor N. (2015) The feasibility of a physical activity program for young adults with Down syndrome: A phase II randomised controlled trial. Journal of Intellectual and Developmental Disability. 40(2): 115-125.

Shogren KA, Burke KM, Antosh AA. (2018) Impact of the self-determined learning model of instruction on self-determination and goal attainment in adolescents with intellectual disability. Journal of Disability Policy Studies.

https://doi.org/10.1177/1044207318792178

Shogren KA. Luckasson R. Schalock RL. (2015) Using context as an integrative framework to align policy goals, supports, and outcomes in intellectual disability. Intellectual \& Developmental Disabilities; 53, 367-376.

Spanos D, Hankey C, Boyle S, Melville C. (2014) Comparing the effectiveness of a multi-component weight loss programme in adults with and without intellectual disabilities. Journal of Human Nutrition \& Dietetics. 27(1): 22-29.

Spanos D, Melville CA, Hankey CR (2013a) Weight management programmesin adults with intellectual disabilities and obesity: A systematic review of the evidence. Nutrition Journal. 12:132.

Spassiani NA, Parker-Harris S. Hammel, J. (2016) Exploring how knowledge translation can improve sustainability of community-based health initiatives for 
people with intellectual/developmental disabilities. Journal of Applied Research in Intellectual Disabilities. 29(5): 433-44.

Stancliffe RJ, Lakin KC, Larson S, et al. (2011) Overweight and obesity among adults with intellectual disabilities who use intellectual disability/developmental disability services in 20 US States. American Journal of Intellectual and Developmental Disabilities.116: 401-418.

Sundblom E, Bergstrom H, Elinder LS. (2015) Understanding the implementation process of a multi-component health promotion programme for adults with intellectual disabilities in Sweden. Journal of Applied Research in Intellectual Disabilities. 28(4): 296-306.

Swinburn B, Sacks G, Hall K. et al. (2011) The global obesity pandemic: shaped by global drivers and local environments. Lancet; 378(9793):804-814.

Taggart L, Brown M, Karatzias T. (2014) Diabetes (Chapter 6). In: Health Promotion for People with Intellectual and Developmental Disabilities (eds L. Taggart and W. Cousins). Open University Press/McGraw-Hill Publishers, Maidenhead, England Taggart L, Cousins W. (eds) (2014) Health Promotion for People with Intellectual and Developmental Disabilities. Open University Press/McGraw-Hill Publishers, Maidenhead, England.

Taggart L, Truesdale-Kennedy M, Coates V. (2013) Management and quality indicators of diabetes mellitus in people with intellectual disabilities. Journal of Intellectual Disability Research: 57(12);1152-1163. 
Tremmel M, Gerdtham UG, Nilsson PM, Saha, S. (2017) Economic burden of obesity: Systematic literature review. International Journal of Environmental Research \& Public Health.14: 435.

Wang Y, Beydoun MA, Liang L, Caballero B, Kumanyika SK. (2008) Will all Americans become overweight or obese? Estimating the progression and cost of the US obesity epidemic. Obesity. 16: 2323-30

Willems M, Hilgenkamp TIM, Havik E, Waninge A, \& Melville CA. (2017) Use of behaviour change techniques in lifestyle change programmesfor people with intellectual disabilities: A systematic review. Research in Developmental Disabilities. 60:256-268.

Willems M, Waninge A, Hilgenkamp TIM. et al. (2018) Effects of lifestyle change programmesfor people with intellectual disabilities: Systematic review and metaanalysis of randomised controlled trials. Journal of Applied Research in Intellectual Disabilities. 31: 949-961.

Wilson, PW, D’Agostino, RB, Sullivan L, Parise H, Kannel WB. (2002) Overweight and obesity as determinants of cardiovascular risk: the Framingham experience. Archives of Internal Medicine. 162(16): 1867-72

World Health Organisation (2004) Global Strategy on Diet, Physical Activity and Health. WHO. Geneva. http://www.who.int/dietphysicalactivity/strategy/eb11344/strat egy_english_web.pdf [accessed 07/10/19]. 
World Health Organisation (2004) The Commission on Ending Childhood Obesity. WHO. Geneva. https://www.who.int/end-childhood-obesity/finalreport/en/ [accessed 22/10/19]. 
Table 1: UK recommended evidence-based obesity management MCls (SIGN, 2010; NICE 2014)

Multi-component: including 600kcal/day energy deficit diets (i.e. containing $600 \mathrm{kcal}$ less than the person needs to stay the same weight), physical activities that fit easily into people's lives (e.g. walking, cycling; and behaviour change methods such as problem-solving and goal setting)

Multi-disciplinary: including input from registered dietitians, psychologists and physical activity instructors

At least 3 months in duration, with sessions that are offered at least weekly or fortnightly, which monitor weight and include a 'weigh-in' at each session

Inclusive of achievable weight loss goals and targets for the individuals

Focused on lifestyle change and the prevention of future weight gain

Inclusive of discussions on how to reduce sedentary behaviour and the types of physical activities that can be incorporated into daily life

Tailored to the individual and provide on-going support 
